
requires working with a large amount of data, so it is necessary to improve the existing parsing techniques. The most asymptotically efficient parsing algorithm that can be applied to any context-free grammar is a matrixbased algorithm proposed by Valiant. This paper aims to present Valiant's algorithm modification, which mai advantage is the possibility to divide the parsing table into successively computed layers of disjoint submatrice where each submatrix of the layer can be processed independently. Moreover, our approach is easily adapted for the string-matching problem. Our evaluation shows that the proposed modification retains all benefits of Valiant's algorithm, especially its high performance achieved by using fast matrix multiplication methods. Also, the modified version decreases a large amount of excessive computations and accelerates the substrings searching.

Keywords: parsing, context-free grammars, matrix operations.

Ю.A. Сусанина, ORCID: 0000-0003-3904-3764<jsusanina@gmail.com>

A.H. Явейн, ORCID: 0000-0001-9733-5429<yaveyn@yandex.ru>

C.B. Григорьев, ORCID: 0000-0002-7966-0698<s.v.grigoriev@spbu.ru> Санкт-Петербургский государственный университет,

199034, Россия, г. Санкт-Петербург, Университетская наб., д. 7/9

Аннотация. Теория формальных языков активно изучается и находит широкое применение во многих областях. Например, в биоинформатике в задачах распознавания и классификации иногда требуется найти подпоследовательности генетических цепочек, обладающие некоторыми характерными чертами, которые могут быть описаны с помошью грамматики. Задача поиска этих подпоследовательностей сводится к проверке их принадлежности некоторому формальному языку, заданному грамматикой При этом часто требуется эффективная обрбол X необход моменг срди алов одним из самых быстрых явяслся алгоритм Валианта, основанный на использовании матричных операций. В данной работе предложен алгоритм, который является модификацией алгоритма Валианта. Его основным достоинством является возможность разбиения матрицы разбора на подслои непересекающихся подматриц, которые могут быть обработаны независимо. Доказана корректность и приведена оценка сложности предложенного алгоритма. Проведенные эксперименты показывают, что он сохранил основные преимущества исходного алгоритма, главное из которых - высокая производительность, полученная за счет использования эффективных методов перемножения матриц. Также предложенный алгоритм позволил заметно уменьшить время, затрачиваемое на поиск подстрок, сократив большое количество избыточных вычислений.

Ключевые слова: синтаксический анализ; контекстно-свободные грамматики; матричные операции

Для цитирования: Сусанина Ю.А., Явейн А.Н., Григорьев С.В. Модификация алгоритма Валианта для Далачи поиска поде уок. Труды ИСП РАН, том 32, вып. 2, 2020 М., стр. 135-148. DOI: 10.15514/ISPRAS2020-32(2)-11

Благодарности: Данная работа выполнена при поддержке гранта РНФ 18-11-00100 и JetBrains Research.

\title{
Modification of Valiant's algorithm for the string-matching problem
}

Y.A. Susanina, ORCID: 0000-0003-3904-3764<jsusanina@gmail.com>

A.N. Yaveyn, ORCID: 0000-0001-9733-5429<yaveyn@yandex ru>

S.V.Grigorev,ORCID:0000-0002-7966-0698<s.v.grigoriev@spbu.ru> Saint Petersburg State University,

7/9, Universitetskaya nab., St. Petersburg, 199034, Russia

Abstract. The theory of formal languages and, particularly, context-free grammars has been extensively studied and applied in different areas. For example, several approaches to the recognition and classification problems in bioinformatics are based on searching the genomic subsequences possessing some specific features which can be described by a context-free grammar. Therefore, the string-matching problem can be reduced to parsing

For citation: Susanina Y.A., Yaveyn A.N., Grigorev S.V. Modification of Valiant's algorithm for the string matching problem. Trudy ISP RAN/Proc. ISP RAS, vol. 32, issue 2, 2020. pp. 135-148 (in Russian). DOI 10.15514/ISPRAS-2020-32(2)-11

Acknowledgements. The research was supported by the Russian Science Foundation grant 18-11-00100 and a grant from JetBrains Research.

\section{1. Введение}

Теория формальных языков активно изучается и находит широкое применение во многих областях [2], прежде всего, в информатике, для описания языков программирования. Также сушествует множество исследований, которые показывают эффективность использования КС-грамматик в биоинформатике $[12,13]$. Так, формальные языки применяются для решения задач распознавания и классификации в биоинформатике, некоторые из которых основань на том, что вторичная структура последовательностей ДНК и РНК содержит в себе важную информацию об организме [16]. Характерные особенности вторичной структуры могут быть описаны с помошью КС-грамматики $[14,15]$, что позволяет свести проблему распознавания и классификации к задаче синтаксического анализа (определения принадлежности некоторой строки к языку, заданному грамматикой).

Большинство алгоритмов синтаксического анализа либо работают за кубическое время (Касами [3], Янгер [4], Эрли [10]), либо применяются только к определенным подклассам КСграмматик (Бернарди, Клауссен [11]). На данный момент одним из самых быстрых алгоритмов, работающих с любой КС-грамматикой, является алгоритм Валианта [5]. Болес того, данный алгоритм был расширен для конъюнктивных и булевых грамматик, которые обладают большей выразительностью, чем КС-грамматики $[1,7,8]$. Однако алгоритм Валианта плохо применим к проблеме нахождения всех подстрок определенной длины, так как он будет выполнять много лишних вызовов перемножения матриц.

В данной работе предложен алгоритм, который является модификацией алгоритма Валианта. За счет изменения порядка вычисления перемножений матриц появилась возможность разбиения матрицы разбора на слои непересекающихся подматриц. Предложенный подход частично решает проблему поиска подстрок за счет простой остановки алгоритма после заполнения определенного слоя. Кроме того, каждая матрица слоя может обрабатываться независимо, что в дальнейшем позволит повысить эффективность алгоритма, используя техники параллельных вычислений. Доказана корректность предложенного алгоритма и приведена оценка сложности. Проведены эксперименты, показывающие, что предложенный алгоритм не проигрывает в производительности алгоритму Валианта и может быть эффективно применен к задаче поиска подстрок.

Работа организована следуюшим образом. В разд. 2 даны основные понятия и приведен исходный алгоритм Валианта; в разд. 3 представлен алгоритм, являюшийся модификацией алгоритма Валианта, легко адаптируемый к задаче поиска подстрок и позволяющий повысить использование параллельных техник, а также доказана корректность и приведена оценка 
сложности модифицированный версии; в разд. 4 показана применимость предложенного нами алгоритма к задаче поиска подстрок; в разд. 5 представлены результаты проведенных экспериментов; заключение и направления будущих исследований приведены в разделе 6 .

\section{2. Обзор}

В этом разделе мы введем основные определения и опишем алгоритм Валианта, на котором основывается предложенная в данной работе модификация.

\section{1 Терминология}

Грамматикой будем называть четверку $(\Sigma, N, R, S)$, где $\Sigma$ - конечное множество терминальных символов, $N$ - конечное множество нетерминальных символов, $R$ - конечное множество правил вида $\alpha \rightarrow \gamma$, где $\alpha \in V^{*} N V^{*}, \gamma \in V^{*}, V=\Sigma \cup N$ и $S \in N$-стартовый символ. Грамматика называется контекстно-свободной (КС), если любое ее правило $r \in R$ имеет вид $A \rightarrow \beta$, где $A \in N, \beta \in V^{*}$.

КС-грамматика $G=(\Sigma, N, R, S)$ называется грамматикой в нормальной форме Хомского, если любое ее правило имеет одну из следующих форм:

- $A \rightarrow B C$,

- $A \rightarrow a$,

- $S \rightarrow \varepsilon$ (если пустая строка $\varepsilon \in L_{G}$ ),

где $A, B, C \in N, a \in \Sigma, L_{G}-$ язык, порождаемый грамматикой $G$.

С помощью $L_{G}(A)$ будем обозначать язык, порождаемый грамматикой $G_{A}=(\Sigma, N, R, A)$.

\section{2 Алгоритм Валианта}

Задачей синтаксического анализа является проверка принадлежности входной строки языку, порождаемому некоторой грамматикой.

Взятый за основу в данной статье алгоритм Валианта относится к табличным методам синтаксического анализа, главная идея которых - построение для входной строки $a=a_{1} \ldots a_{n}$ и КС-грамматики в нормальной форме Хомского $G=\left(\sum, N, R, S\right)$ таблицы (далее - матрицы) разбора $T$ размера $(n+1) \times(n+1)$, где $T_{i, j}=\left\{A: A \in N, a_{i+1} \ldots a_{j} \in\right.$ $\left.L_{G}(A)\right\}$ для всех $i<j$.

Элементы матрицы $T$ должны заполняться последовательно, начиная с диагонали: $T_{i-1, i}=\left\{A: A \rightarrow a_{i} \in R\right\}$.

Затем $T_{i, j}$ вычисляются по формуле $T_{i, j}=f\left(P_{i, j}\right)$, где

$$
\begin{gathered}
P_{i, j}=\bigcup_{k=i+1}^{j-1} T_{i, k} \times T_{k, j}, \\
f\left(P_{i, j}\right)=\left\{A \mid \exists A \rightarrow B C \in R,(B, C) \in P_{i, j}\right\} .
\end{gathered}
$$

Входная строка $a=a_{1} \ldots a_{n}$ принадлежит языку $L_{G}$ тогда и только тогда, когда $S \in T_{0, n}$. Если все элементы матрицы $T$ заполнять последовательно, то вычислительная сложность данного алгоритма будет равна $O\left(n^{3}\right)$. Наиболее затратной по времени операцией является вычисление $\bigcup_{k=i+1}^{j-1} T_{i, k} \times T_{k, j}$. Валиант предложил реорганизовать порядок заполнения элементов матрицы разбора так, что стало возможным выполнить эти вычисления как перемножение некоторого количества булевых матриц.

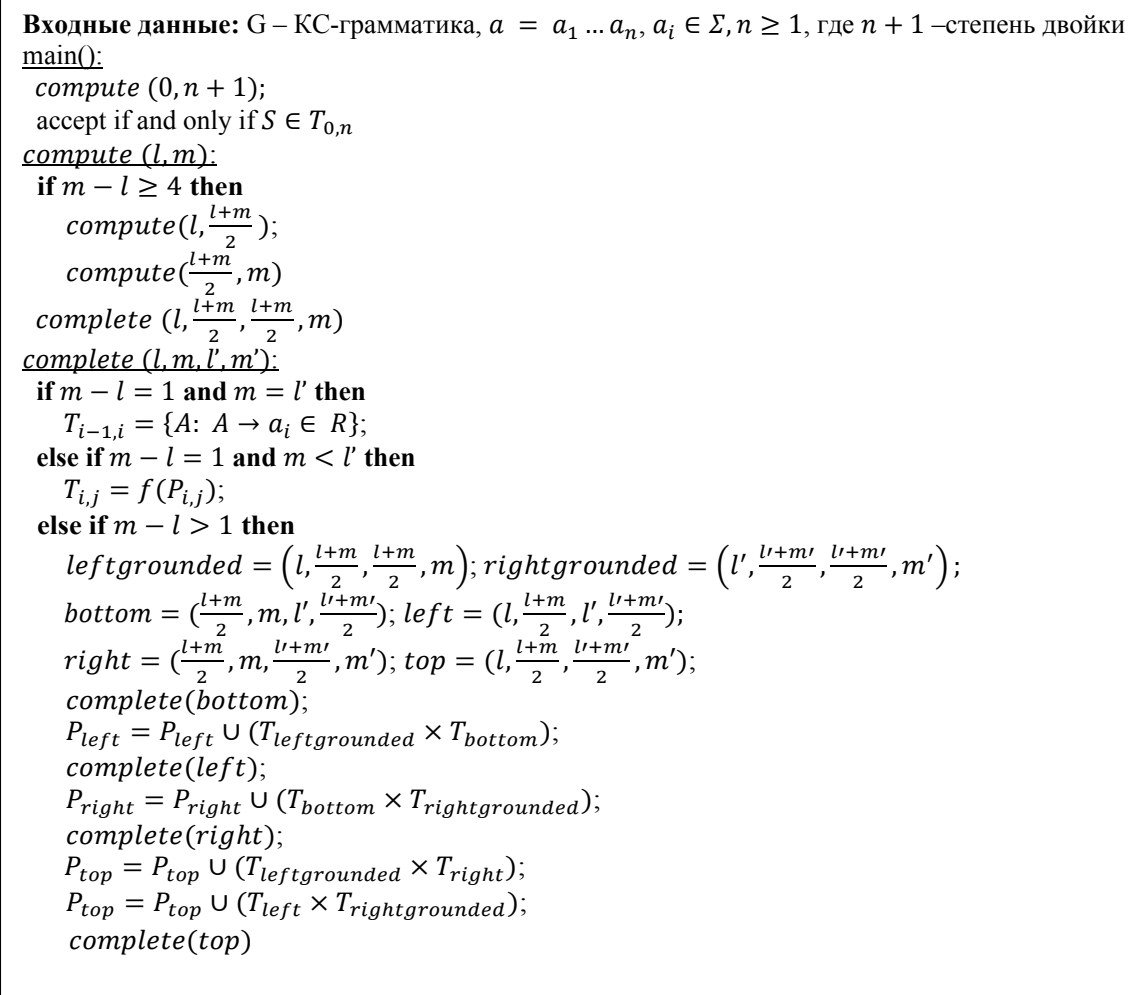

Листинг 1. Алгоритм Валианта

Listing 1. Valiant's algorithm

Сначала введем понятие перемножения двух подматриц матрицы разбора $T$.

Пусть $X \in\left(2^{N}\right)^{m \times l}, Y \in\left(2^{N}\right)^{l \times n}-$ две подматрицы $T$, тогда $X \times Y=Z$, где $Z \in\left(2^{N}\right)^{m \times n}$ и $Z_{i, j}=\bigcup_{k=1}^{l} X_{i, k} \times Y_{k, j}$.

Теперь можно представить $X \times Y=Z$ как перемножение $|N|^{2}$ булевых матриц (для каждой пары нетерминалов). Определим матрицу, соответствующую паре нетерминалов $(B, C)$, как $Z^{(B, C)} \cdot Z_{i, j}^{(B, C)}=1$ тогда и только тогда, когда $(B, C) \in Z_{i, j}$. Также заметим, что $Z^{(B, C)}=$ $X^{B} \times Y^{C}$. Более того, каждое из перемножений булевых матриц может быть обработано независимо.

С этими изменениями сложность алгоритма составляет $O(B M M(n) \log (n))$, где $B M M(n)-$ время, необходимое для перемножения двух булевых матриц размера $n \times n$.

Алгоритм Валианта представлен на листинге 1 . Все элементы матриц $T$ и $P$ инициализируются пустыми множествами. Затем эти элементы последовательно заполняются двумя рекурсивными процедурами

Процедура compute $(l, m)$ корректно заполняет все $T_{i, j}$ для всех $l \leq i<j<m$.

Процедура complete $\left(l, m, l^{\prime}, m^{\prime}\right)$ заполняет все $T_{i, j}$ для всех $l \leq i<m$ и $l^{\prime} \leq j<m^{\prime}$. Для корректной работы этой процедуры, во-первых, необходимо, чтобы элементы $T_{i, j}$ для всех $i$ и $j$, таких что $l \leq i<j \leq m$ и $l^{\prime} \leq i<j \leq m^{\prime}$ уже были построены. Во-вторых, 
текущее значение $P_{i, j}$ для всех $i$ и $j$, таких что $l \leq i<m$ и $l^{\prime} \leq j<m^{\prime}$, должно быть следующим:

$$
P_{i, j}=\left\{(B, C): \exists k,\left(m \leq k<l^{\prime}\right), a_{i+1} \ldots a_{k} \in L_{G}(B), a_{k+1} \ldots a_{j} \in L_{G}(C)\right\} .
$$

Процесс разбиения матрицы во время выполнения процедуры complete $\left(l, m, l^{\prime}, m^{\prime}\right)$

показан на рис. 1. Матрица в данном случае задана четверкой $\left(l, m, l^{\prime}, m^{\prime}\right)$, и она делится на четыре равные квадратные подматрицы: bottom $=\left(\frac{l+m}{2}, m, l^{\prime}, \frac{l+m}{2}\right)$, left $=$ $\left(l, \frac{l+m}{2}, l^{\prime}, \frac{l^{\prime+m} \prime}{2}\right), r i g h t=\left(\frac{l+m}{2}, m, \frac{l^{\prime+m}}{2}, m^{\prime}\right)$, top $=\left(l, \frac{l+m}{2}, \frac{l++m^{\prime}}{2}, m^{\prime}\right)$. Кроме того, на рис.1 изображены две подматрицы, использующиеся в перемножении: leftgrounded = $\left(l, \frac{l+m}{2}, \frac{l+m}{2}, m\right)$, rightgrounded $=\left(l^{\prime}, \frac{l++m^{\prime}}{2}, \frac{l^{\prime+m} \prime}{2}, m^{\prime}\right)$.

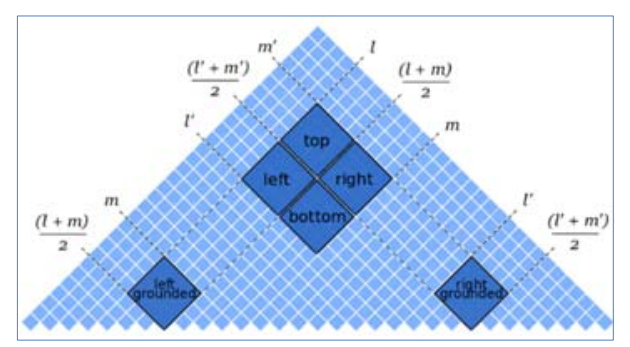

Рис. 1. Разбиение матрии, использованное в процедуре сотрlete $\left(l, m, l^{\prime}, m^{\prime}\right)$ Fig. 1. Matrix partition used in complete $\left(l, m, l^{\prime}, m^{\prime}\right)$ procedure

На рис. 2 представлено начало процесса заполнения матрицы разбора. В следующем разделе будет приведен пример работы модификации, который наглядно продемонстрирует преимущества предложенной нами версии.

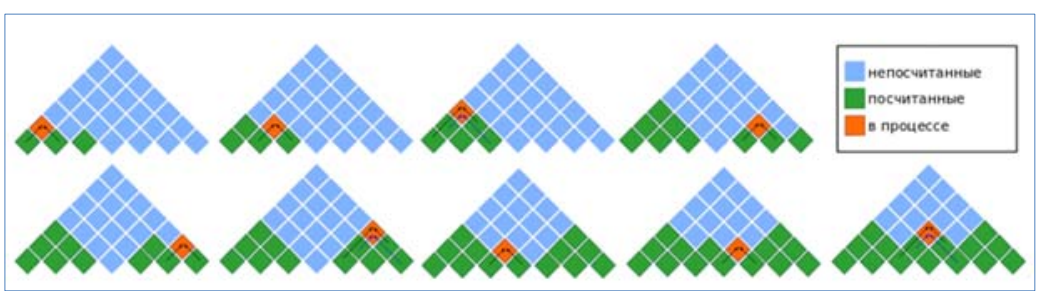

\section{Рис. 2. Пример работы алгоритма Валианта}

Fig. 2. An example of Valiant's algorithm

\section{3 Задача поиска подстрок}

При анализе генетических последовательностей в биоинформатике часто возникает задача нахождения одной или нескольких подпоследовательностей,

обладающих какими-либо характерными чертами. Наличие этих подпоследовательностей, а также их взаимное расположение позволяют получить важную информацию об исследуемом организме, например, о его происхождении. Часть подходов, используемых для их поиска, основаны на формальных грамматиках, с помощью которых можно эффективно описать эти черты. Главным недостатком таких подходов являются существенные проблемы с производительностью [16], которые можно решить с помощью алгоритма Валианта, но его трудно остановить на определенном этапе заполнения матрицы разбора и это потребует много лишних перемножений матриц.
Susanina Y.A., Yaveyn A.N., Grigorev S.V. Modification of Valiant's algorithm for the string matching problem. Trudy ISP RAN/Proc. ISP

\section{3. Модификация алгоритма Валианта}

В данном разделе мы представляем алгоритм, являющийся модификацией алгоритма Валианта. За счет изменения порядка вычисления подматриц предложенный алгоритм обладает такими практическими преимуществами, как возможность применения для решения задачи поиска подстрок и упрощение использования параллельных вычислений.

Главное отличие предложенного алгоритма - это возможность разделения матрицы разбора на слои непересекающихся подматриц одинакового размера. Пример разбиения матриц на такие слои представлен на рис. 3. Каждый слой состоит из квадратных подматриц, размер которых равен $2^{n}$, где $n>0$. Слои заполняются последовательно, снизу вверх, и каждая матрица слоя может обрабатываться независимо. На рис. 3 каждый слой выделен отдельным цветом, нижняя подматрица матрицы слоя (bottom), принадлежит предыдущему слою и уже заполнена. Таким образом, для каждой матрицы слоя нам необходимо заполнить только left,right и top-подматрицы, поэтому такие слои мы будем называть V-образными.

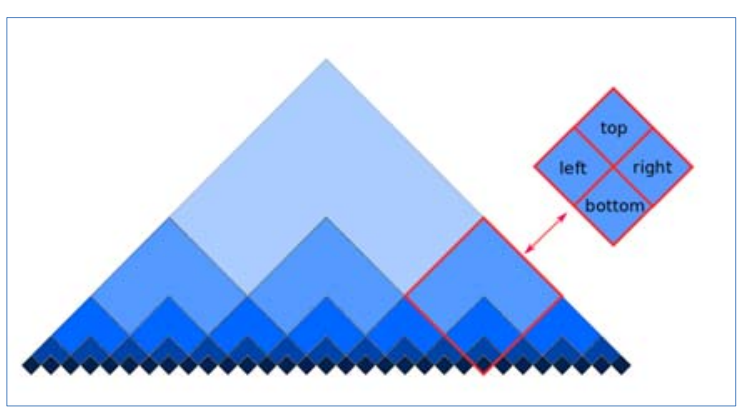

Рис. 3. Деление матрии на V-образные слои Fig. 3. Matrix partition on V-shaped layers

Пример работы алгоритма показан на рис. 4. Нижний слой, состоящий из подматриц размера 1, вычисляется заранее, а заполнение матрицы начинается со второго слоя. (Здесь и далее, под слоем матриц будем понимать некоторое множество её подматриц разбора.) Также на рис. 4 на каждом шаге изображены операции, которые могут быть выполненные независимо, что позволяет значительно упростить разработку параллельной версии алгоритма.

Модификация алгоритма Валианта представлена на листинге 1. Процедура main () заполняет нижний слой матрицы $\left(T_{l l+1}\right)$, а затем разделяет матрицу на слои так, как было описано ранее, и эти слои корректно вычисляются в процедуре completeVLayer (). Таким образом, вызов функции main () заполнит всю матрицу разбора и вернет информацию о выводимости строки для заданной грамматики.

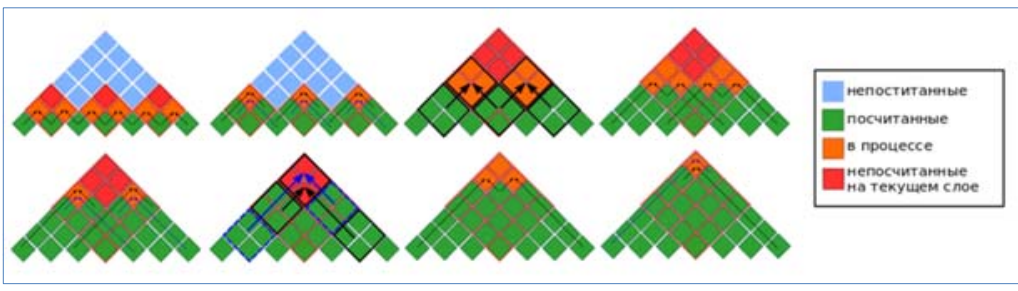

Рис. 4. Пример работы модификации алгоритма Валианта Fig. 4. An example of the modification of Valiant's algorithm 


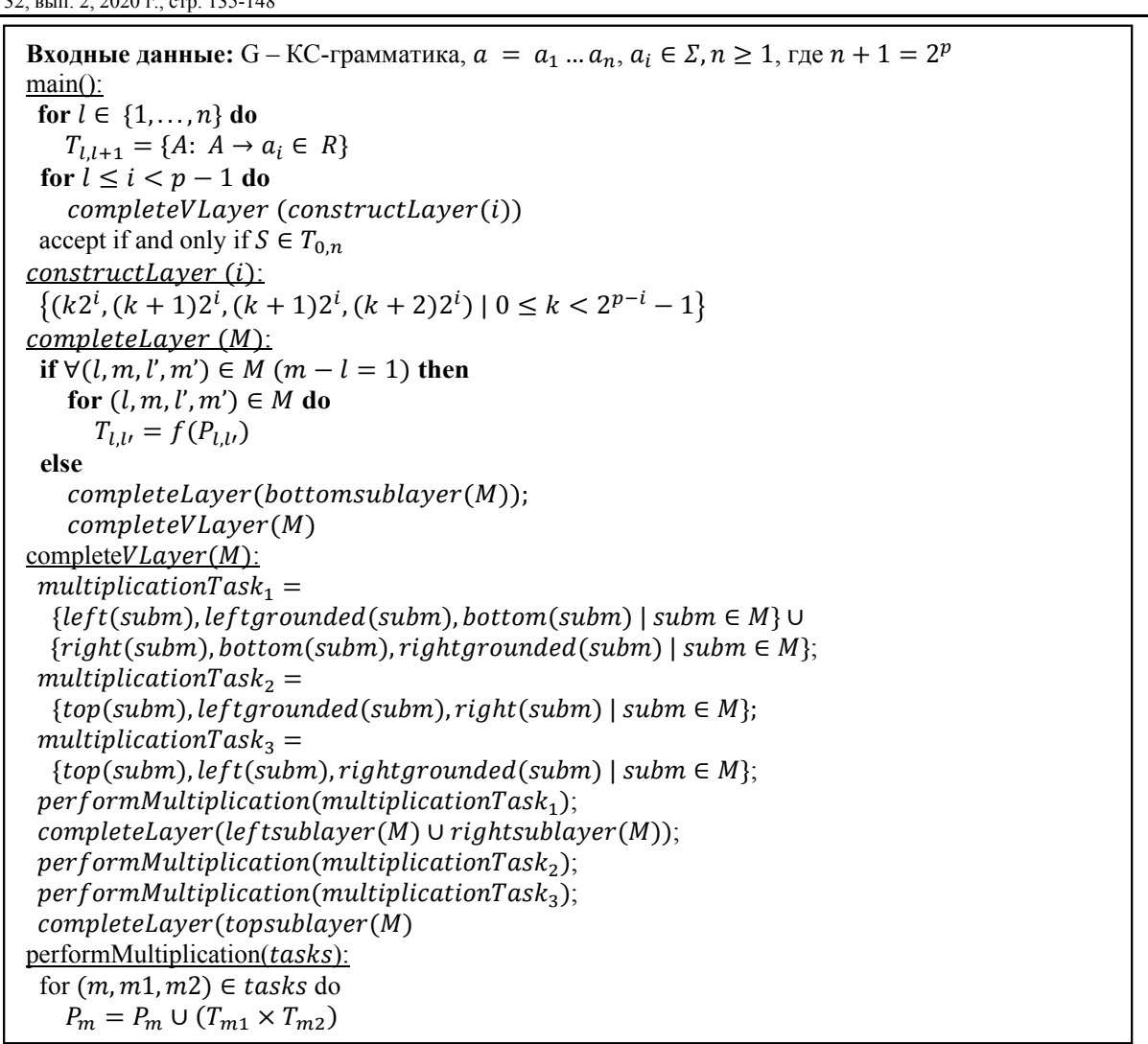

Листинг 2. Модификация алгоритма Валианта

Listing 2. Modification of Valiant's algorithm

Для краткости, аналогично тому, как сделано в алгоритме Валианта, определим дополнительные функции: left(subm), right(subm), bottom(subm), top(subm), rightgrounded(subm) and leftgrounded(subm), которые возвращают различные подматрицы для матрицы $s u b m=\left(l, m, l^{\prime}, m^{\prime}\right)$. Также определим функции для слоя подматриц $M$ :

- $\operatorname{bottomsublayer}(M)=\{$ bottom $(\operatorname{subm}) \mid \operatorname{subm} \in M\}$,

- leftsublayer $(M)=\{$ left(subm) $\mid \operatorname{subm} \in M\}$,

- $\operatorname{rightsublayer}(M)=\{\operatorname{right}(\operatorname{subm}) \mid \operatorname{subm} \in M\}$,

- $\operatorname{topsublayer}(M)=\{\operatorname{top}(\operatorname{subm}) \mid \operatorname{subm} \in M\}$

Процедура completeVLayer $(M)$ принимает на вход слой непересекающихся подматриц одинакового размера $M$. Для каждой $s u b m=\left(l, m, l^{\prime}, m^{\prime}\right) \in M$ эта процедура вычисляе left(subm), right(subm), top(subm). Для корректной работы этой процедуры, вопервых, необходимо, чтобы элементы bottom (subm) и $T_{i, j}$ для всех $i$ и $j$, таких что $l \leq$ $i<j \leq m$ и $l^{\prime} \leq i<j \leq m^{\prime}$, уже были построены. Во-вторых, текущее значение $P_{i, j}$ для всех $i$ и $j$, таких что $l \leq i<m$ и $l^{\prime} \leq j<m^{\prime}$, должно быть следующим: $P_{i, j}=\left\{(B, C): \exists k,\left(m \leq k<l^{\prime}\right), a_{i+1} \ldots a_{k} \in L_{G}(B), a_{k+1} \ldots a_{j} \in L_{G}(C)\right\}$.
Процедура completeLayer $(M)$ также принимает на вход набор подматриц $M$, но каждую subm $\in M$ заполняет полностью.Ограничения на входные данные у этой процедуры такие же, как и у процедуры completeVLayer(), за исключением ограничения на bottom(subm), которое в данном случае не требуется.

Другими словами, completeVLayer $(M)$ вычисляет весь $V$-образный слой $M$, а completeLayer $\left(M_{2}\right)$ - вспомогательная функция, необходимая для вычисления меньших квадратных подматриц слоя $M$.

Наконец процедура performMultiplication(tasks), где tasks - это массив троек подматриц, реализует основной шаг алгоритма - перемножение матриц. Стоит заметить, что в отличие от исходного алгоритма Валианта, в данном случае $\mid$ tasks $\mid \geq 1$ и каждый task $\in$ tasks может быть выполнен параллельно.

Для представленного алгоритма докажем следующие утверждения.

Лемма 1. Пусть $M$ - слой и для всех $\left(l, m, l^{\prime}, m^{\prime}\right) \in M$ справедливо следующее:

- для всех $i$ и $j$, таких что $l \leq i<j \leq m$ и $l^{\prime} \leq i<j \leq m^{\prime}$,

$$
T_{i, j}=\left\{A: \quad a_{i+1} \ldots a_{j} \in L_{G}(A)\right\} ;
$$

- $\quad$ для всех $i$ и $j$, таких что $l \leq i<m$ и $l^{\prime} \leq j<m^{\prime}$,

$$
P_{i, j}=\left\{(B, C): \exists k,\left(m \leq k<l^{\prime}\right), a_{i+1} \ldots a_{k} \in L_{G}(B), a_{k+1} \ldots a_{j} \in L_{G}(C)\right\} .
$$

Тогда процедура completeLayer $(M)$ возвращает корректно заполненные $T_{i, j}$ для всех $i$ и $j$, таких что $l \leq i<m$ и $l^{\prime} \leq j<m^{\prime}$ при этом $\left(l, m, l^{\prime}, m^{\prime}\right) \in M$.

\section{Доказательство.}

Индукция по размеру матриц в слое М.

Теорема 1. (Корректность алгоритма). Алгоритм, представленный на листинге 2 , корректно заполняет $T_{i, j}$ для всех $i$ и $j$, и входная строка $a_{1} \ldots a_{n} \in L_{G}(S)$ тогда и только тогда, когда $S \in T_{0, n}$.

\section{Доказательство.}

Перед тем, как доказать утверждение теоремы, докажем по индукции, что все слои матрицы разбора $T$ вычисляются корректно.

База индукиии. Слой размера $1 \times 1$ корректно заполняется в строках 2-3 листинга 2. Индукиионный переход. Предположим, что все слои размера $\leq 2^{p-2} \times 2^{p-2}$ вычислены корректно.

Обозначим слой размера $2^{p-1} \times 2^{p-1}$ как $M$. Будем рассматривать одну матрицу слоя $\mathrm{subm}=\left(l, m, l^{\prime}, m^{\prime}\right)$, так как заполнение остальных подматриц производится аналогично. Рассмотрим вызов процедуры completeVLayer $(M)$. Заметим, что все $T_{i, j}$ для всех $i$ и $j$, таких что $l \leq i<j<m$ и $l^{\prime} \leq i<j<m^{\prime}$, уже корректно заполнены, так как эти элементы лежат в слоях, которые уже вычислены по индукционному предположению. В начале выполнения процедуры completeVLayer $(M)$, performMultiplication (multiplicationTask ${ }_{1}$ ) добавляет $\quad$ к каждому $P_{i, j}$ все парь нетерминалов $(B, C)$, такие что $\exists k,\left(\frac{l+m}{2} \leq k<l^{\prime}\right), a_{i+1} \ldots a_{k} \in L_{G}(B), a_{k+1} \ldots a_{j} \in L_{G}(C)$ для всех $(i, j) \in$ leftsublayer $(M)$ и все пары $(B, C)$, такие что $\exists k,\left(m \leq k<\frac{l++m \prime}{2}\right), a_{i+1} \ldots a_{k} \in$ $L_{G}(B), a_{k+1} \ldots a_{j} \in L_{G}(C)$ для всех $(i, j) \in$ rightsublayer $(M)$. Теперь все предусловия для вызова процедуры completeLayer (leftsublayer $(M)$ U rightsublayer $(M))$ выполнены и она вернет корректно заполненные leftsublayer $(M) \cup$ rightsublayer $(M)$.

Затем функция performMultiplication вызывается от аргументов multiplicationTask ${ }_{2}$ и multiplicationTask ${ }_{3}$ и к каждому $P_{i, j}$ добавляет пары нетерминалов $(B, C)$, такие что $\exists k,\left(\frac{l+m}{2} \leq k<m\right), a_{i+1} \ldots a_{k} \in L_{G}(B), a_{k+1} \ldots a_{j} \in L_{G}(C)$ и $(B, C)$, такие что $\exists k,\left(l^{\prime} \leq k<\right.$ 
Таким образом, completeVLayer $(M)$ для каждого слоя $M$ возвращает корректные $T_{i, j}$ для всех $(i, j) \in M$ матрицы $T$ и строки 4-6 листинга 2 возвращают все $T_{i, j}=\{A: A \in N$, $\left.a_{i+1} \ldots a_{j} \in L_{G}(A)\right\}$.

Лемма 2. Пусть calls $s_{i}$ - это количество вызовов процедуры completeVLayer $(M)$, где для всех $\left(l, m, l^{\prime}, m^{\prime}\right) \in M$ выполнено $m-l=2^{p-i}$. Тогда истинны следующие утверждения:

- $\quad$ для всех $i \in\{1, \ldots, p-1\} \sum_{n=1}^{\text {calls }_{i}}|M|=2^{2 i-1}-2^{i-1}$;

- для всех $i \in\{1, \ldots, p-1\}$ матрицы размера $2^{p-i} \times 2^{p-i}$ перемножаются ровно $2^{2 i-1}-$ $2^{i}$ раз.

\section{Доказательство.}

Сначала докажем первое утверждение индукцией по $i$.

База индукции. При $i=1$ : calls $_{1}=1$ и $|M|=1$. Следовательно, $2^{2 i-1}-2^{i-1}=2^{1}-$ $2^{0}=1$.

Индукиионный переход. Предположим, что $\sum_{n=1}^{\text {calls }_{i}}|M|=2^{2 i-1}-2^{i-1}$ для всех $i \in\{1, \ldots, j\}$. Пусть $i=j+1$.

Заметим, что функция constructLayer $(i)$ возвращает $2^{p-i}-1$ матриц размера $2^{i} \times 2^{i}$, то есть при вызове процедуры completeVLayer (constructLayer $(k-i)$ ) процедура constructLayer $(k-i)$ вернет $2^{i}-1$ матриц размера $2^{p-i} \times 2^{p-i}$. Также, completeVLayer $(M)$ будет вызван 3 раза для левых, правых и верхних подматриц матриц размера $2^{p-(i-1)} \times 2^{p-(i-1)}$. Кроме того, completeVLayer $(M)$ вызывается 4 раза для нижних, левых, правых и верхних подматриц матриц размера $2^{p-(i-2)} \times 2^{p-(i-2)}$, за исключением левых, правых и верхних подматриц размера $2^{i-2}-1$ матриц, которые к этому моменту уже были посчитаны.

Таким образом, $\sum_{n=1}^{\text {calls }}|M|=2^{i}-1+3 *\left(2^{2(i-1)-1}-2^{(i-1)-1}\right)+4 *\left(2^{2(i-2)-1}-\right.$ $\left.2^{(i-2)-1}\right)-\left(2^{i-2}-1\right)=2^{2 i-1}-2^{i-1}$.

Теперь мы знаем, что $\sum_{n=1}^{\text {calls }_{i-1}}|M|=2^{2(i-1)-1}-2^{(i-1)-1}$, и можем доказать второе утверждение. Посчитаем количество перемножений матриц размера $2^{p-i} \times 2^{p-i}$. Функция performMultiplication вызывается 3 раза, $\mid$ multiplicationTask $\mid=2 *\left(2^{2(i-1)-1}-\right.$ $\left.2^{(i-1)-1}\right)$ и $\mid$ multiplicationTask $k_{2}|=|$ multiplicationTask $3 \mid=2^{2(i-1)-1}-2^{(i-1)-1}$. То есть, количество перемножений подматриц размера $2^{p-i} \times 2^{p-i}$ равно $4 *\left(2^{2(i-1)-1}-\right.$ $\left.2^{(i-1)-1}\right)=2^{2 i-1}-2^{i}$

Теорема 2. (Оиенка сложности алгоритма). Пусть $|G|$ - длина описания грамматики $G$ и $n$ - длина входной строки. Тогда алгоритм, представленный на листинге 2 , заполняет матрицу $T$ за $O(|G| B M M(n) \log (n))$, где $B M M(n)$ - время, необходимое для перемножения двух булевых матриц размера $n \times n$.

\section{Доказательство.}

Так как в лемме 2 было показано, что количество перемножений матриц не изменилось по сравнению с исходной версией алгоритма Валианта, то доказательство будет идентично доказательству теоремы 1 в [8].

Таким образом, мы доказали корректность предложенного алгоритма, а также показали, что его сложность осталась такой же, как сложность исходного алгоритма Валианта.

\section{4. Применение алгоритма к задаче почска подстрок}

В данном разделе мы продемонстрируем, как предложенный алгоритм может быть применена к задаче поиска подстрок.

Пусть для входной строки размера $n=2^{p}$ мы хотим найти все подстроки размера $s$, которые принадлежат языку, заданному грамматикой $G$. Тогда мы должны посчитать слои подматриц, размер которых не превышает $2^{r}$, где $2^{r-2}<s \leq 2^{r-1}$.

Пусть $r=p-(m-2)$ и, следовательно, $m-2=p-r$.

Для всех $m \leq i \leq p$ перемножение матриц размера $2^{p-i} \times 2^{p-i}$ выполняется ровно $2^{2 i-1}-$ $2^{i}$ раз и каждое из них включает перемножение $C=O(|G|)$ булевых подматриц.

$$
\begin{aligned}
& C * \sum_{i=m}^{p} 2^{2 i-1} * 2^{\omega(p-i)} * f\left(2^{p-i}\right)=C * 2^{\omega r} * \sum_{i=2}^{r} 2^{(2-\omega) i} * 2^{2(p-r)-1} * f\left(2^{r-i}\right) \\
& \leq C * 2^{\omega r} * f\left(2^{r}\right) * 2^{2(p-r)-1} * \sum_{i=2}^{r} 2^{(2-\omega) i}=B M M\left(2^{r}\right) * 2^{2(p-r)-1} * \sum_{i=2}^{r} 2^{(2-\omega) i}
\end{aligned}
$$

Временная сложность алгоритма для поиска всех подстрок длины $s$ равна $O\left(2^{2(p-r)-1}|G| B M M\left(2^{r}\right) \log (n)\right)$, где дополнительный множитель обозначает количество матриц в последнем вычисленном слое. Однако этот множитель, во-первых, мал относительно общей по времени работы алгоритма, во-вторых, не существенен, так как эти матрищы могут быть обработаны параллельно. Алгоритм Валианта, в отличие от модификации, не может так легко быть применен к данной задаче. В нем будет необходимо полностью вычислить, как минимум, две треугольные подматрицы размера $\frac{n}{2}$ (как показано на рис. 5). Это значит, что в любом случае не получится добиться сложности лучше, чем $O\left(|G| B M M\left(2^{p-1}\right) \log (p-2)\right)$.

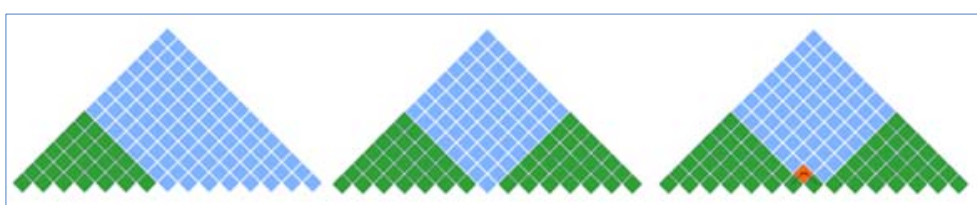

Рис. 5. Количество элементов, вычисляемых в алгоритме Валианта (2 треугольные подматриць размера $\frac{n}{2}$, выделенные зеленым иветом)

Fig. 5. Elements computed in Valiant's algorithm (at least 2 triangle submatrices of size $\frac{n}{2}$, which are marked in green).

В завершение данного раздела скажем, что предложенный алгоритм может быть эффективно применен для строк размера $s \ll n$, что и было показано в проведённых экспериментах

\section{5. Эксперименть}

В этом разделе мы приводим результаты экспериментов, целью которых является демонстрация практической применимости предложенного алгоритма к задаче поиска подстрок

\section{1 Постановка экспериментов}

Эксперименты проводились на рабочей станции со следующими характеристиками: операционная система - Linux Mint 19.1, ЦПУ — Intel i5-8250U, 1600-3400 Mhz, 4 Core(s), 8 Logical Processor(s), оперативная память - 8 GB. 

языке программирования С++. Для перемножения подматриц использовалась библиотека для высокоэффективной работы с булевыми матрицами M4RI [9].

Основной целью поставленных экспериментов было исследование эффективности предложенного алгоритма.

Для этого были поставлены следующие вопросы:

RQ1. Сравнение предложенного алгоритма с исходным алгоритмом Валианта.

RQ2. Эффективность применения предложенного алгоритма для задачи поиска подстрок. Для сравнительного анализа алгоритмы были реализованы в соответствии с листингами 1 и 2. При исследовании эффективности предложенного алгоритма к задаче поиска подстрок была изменена функция $\operatorname{main}()$ из листинга 2: теперь она принимает дополнительный аргумент $s$ - длину максимальной искомой подстроки и вычисляет только те слои, которые содержат в себе нужные подстроки, как было показано в предыдущем разделе.

Для экспериментов использовалась КС-грамматика $D_{2}$, порождающая язык Дика с двумя видами скобок и стартовым нетерминалом $\mathrm{S}$ [17]. Правила данной грамматики

представлены на рис. 6. [Привлеките внимание читателя с помощью яркой цитаты из

документа или используйте это место, чтобы выделить ключевой момент. Чтобы поместить это текстовое поле в любой части страницы, просто перетащите его.]

\section{$S \rightarrow S S|(S)|[S] \mid \varepsilon$}

Рис. 6. Грамматика $D_{2}$

Fig. 6. Grammar $D_{2}$

Эта грамматика была выбрана потому, что грамматики для описания правильных скобочных последовательностей часто применяются при анализе строк в биоинформатике.

Грамматика $D_{2}$ переводится в нормальную форму Хомского и подается на вход алгоритму со специально сгенерированными строками различной длины (127-8191 символов). Строки составлены следующим образом: заранее создается подстрока, принадлежащая языку Дика, далее в полную строку вставляется максимально возможное количество созданных подстрок, которые можно разделить "перегородками" (терминалами, из-за которых все остальные строки, кроме вставленных, будут невыводимыми в грамматике $D_{2}$ ). Строки были созданы таким образом, чтобы проверять корректность предложенного алгоритма.

\section{2 Анализ результатов}

RQ1. Сравнение предложенного алгоритма с исходным алгоритмом Валианта.

Результаты сравнительного анализа алгоритма Валианта и его модификации представлены в табл. 1, где $N$ - длина сгенерированной строки. Для двух реализаций - алгоритма Валианта (VAL) и предложенного алгоритма (MOD) представлено время работы в миллисекундах.

Результаты сравнительного анализа (см. табл. 1) показывают, что предложенная модификация и исходный алгоритм Валианта работают практически одинаково.

RQ2. Эффективность применения предложенного алгоритма для задачи поиска подстрок

Результаты работы адаптированной к задаче поиска подстрок модификации представлены в табл. 2, где $N$ - длина сгенерированной строки, $\mathrm{s}$ - длина искомых подстрок. Для алгоритма Валианта (VAL) и модификации с измененной функцией $\operatorname{main}()$ (ADP) представлено время работы в миллисекундах.

Табл. 1. Результаты сравнительного анализа

Table 1. Evaluation results for comparative analysis

\begin{tabular}{|c|c|c|}
\hline $\mathbf{N}$ & VAL & MOD \\
\hline $\mathbf{1 2 7}$ & 78 & 76 \\
\hline $\mathbf{2 5 5}$ & 289 & 292 \\
\hline $\mathbf{5 1 1}$ & 1212 & 1177 \\
\hline $\mathbf{1 0 2 3}$ & 4858 & 4779 \\
\hline $\mathbf{2 0 4 7}$ & 19613 & 19279 \\
\hline $\mathbf{4 0 9 5}$ & 78361 & 78279 \\
\hline $\mathbf{8 1 9 1}$ & 315677 & 315088 \\
\hline
\end{tabular}

Табл. 2. Результаты работы алгоритма для задачи поиска подстрок Table 2. Evaluation results for string-matching problem

\begin{tabular}{|c|c|c|c|}
\hline $\mathbf{S}$ & $\mathbf{N}$ & VAL & ADP \\
\hline $\mathbf{2 5 0}$ & $\mathbf{1 0 2 3}$ & 4858 & 2996 \\
\hline $\mathbf{2 5 0}$ & $\mathbf{2 0 4 7}$ & 19613 & 6649 \\
\hline $\mathbf{5 1 0}$ & $\mathbf{2 0 4 7}$ & 19613 & 12178 \\
\hline $\mathbf{2 5 0}$ & $\mathbf{4 0 9 5}$ & 78361 & 13825 \\
\hline $\mathbf{5 1 0}$ & $\mathbf{4 0 9 5}$ & 78361 & 26576 \\
\hline $\mathbf{1 0 2 0}$ & $\mathbf{4 0 9 5}$ & 78361 & 48314 \\
\hline $\mathbf{2 5 0}$ & $\mathbf{8 1 9 1}$ & 315677 & 28904 \\
\hline $\mathbf{5 1 0}$ & $\mathbf{8 1 9 1}$ & 315677 & 56703 \\
\hline $\mathbf{1 0 2 0}$ & $\mathbf{8 1 9 1}$ & 315677 & 108382 \\
\hline $\mathbf{2 0 4 0}$ & $\mathbf{8 1 9 1}$ & 315677 & 197324 \\
\hline
\end{tabular}

Результаты второго эксперимента (см. табл. 2) показывают, что адаптированная версия модификации может быть эффективно применена к задаче поиска подстрок: она корректно находит все выводимые подстроки в строке и работает существенно быстрее алгоритма Валианта, который совершает большое количество лишних вычислений из-за сложности его преждевременной остановки.

Таким образом, поставленные эксперименты демонстрируют практическую применимость предложенного алгоритма.

\section{6. Заключение}

В данной работе был предложен алгоритм, являющейся модификацией алгоритма Валианта, который обладает некоторыми преимуществами по сравнению с исходной версией. За счет разбиения исходной матрицы на слои подматриц появилась возможность останавливать работу алгоритма, не вычисляя всю матрицу разбора до конца, если этого требует поставленная задача. Проведенные эксперименты показали, что предложенный алгоритм не проигрывает в производительности исходной версии алгоритма. Более того, была показана применимость данной модификации к задаче поиска подстрок.

Определим несколько направлений для будущих исследований. Так как все подматрицы в слое могут быть обработаны независимо, необходимо проверить, насколько эффективно могут быть применены техники параллельных вычислений. Кроме того, открытым остается вопрос, можно ли как-нибудь еще изменить порядок перемножения подматриц, чтобы полностью избавить алгоритм от рекурсивных вызовов? 


\section{Список литературы / References}

[1]. Okhotin A. Conjunctive grammars. Journal of Automata, Languages and Combinatorics, vol. 6, no. 4 2001, pp. 519-535.

[2]. Chomsky N. On certain formal properties of grammars. Information and control, vol. 2, no. 2, 1959, pp. 137-167.

[3]. Kasami T. An efficient recognition and syntax analysis algorithm for context-free languages. Technical Report AFCRL-65-758, Air Force Cambridge Research Laboratory, 1965.

[4]. Younger D.H. Recognition and parsing of context-free languages in time n3. Information and control, vol. 10, no. 2, 1967, pp. 189-208.

[5]. Valiant L.G. General context-free recognition in less than cubic time. Journal of computer and system sciences, vol. 10, no. 2, 1975, pp. 308-315.

6]. Okhotin A. Conjunctive and Boolean grammars: the true general case of the context-free grammars. Computer Science Review, vol. 9, 2013, pp. 27-59.

[7]. Okhotin A. Boolean grammars. Information and Computation, vol. 194, issue 1, 2004, pp. 19-48.

[8]. Okhotin A. Parsing by matrix multiplication generalized to Boolean grammars. Theoretical Computer Science, vol. 516, 2014, pp. 101-120.

[9]. Albrecht M., Bard G. The M4RI Library. The M4RI Team. 2019. Available at: https://bitbucket.org/malb/m4ri, accessed 20.01.2010.

[10]. Earley J. An effcient context-free parsing algorithm. Communications of the ACM, vol. 13, no. 2, 1970 , pp. $94-102$

[11]. Bernardy J.P., Claessen K. Effcient divide-and-conquer parsing of practical context-free languages. ACM SIGPLAN Notice, vol. 48, no. 9, 2013, pp.111-122

[12]. Grigorev S., Lunina P. The Composition of Dense Neural Networks and Formal Grammars for Secondary Structure Analysis. In Proc. of the 12th International Joint Conference on Biomedical Engineering Systems and Technologies, vol. 3, 2019, pp. 234-241.

[13]. Rivas E., Eddy S.R. The language of RNA: a formal grammar that includes pseudoknots. Bioinformatics, vol. 16, no. 4, 2000, pp. 334-340.

[14]. Knudsen B. and Hein J. Rna secondary structure prediction using stochastic context-free grammars and evolutionary history. Bioinformatics, vol. 15, no. 6, 1999, pp. 446-454

[15]. Dowell R.D., Eddy S.R. Evaluation of several lightweight stochastic context-free grammars for rna secondary structure prediction. BMC bioinformatics, vol. 5, no. 1, 2004, Article number: 71.

[16]. Durbin R., Eddy S., Krogh A., and Mitchison G. Biological sequence analysis. Cambridge University Press, 1996, $356 \mathrm{p}$

[17]. Hopcroft J.E., Ullman J.D. Formal languages and their relation to automata. Addison-Wesley, 1969, 242 p.

\section{Информация об авторах / Information about authors}

Юлия Алексеевна СУСАНИНА получила степень бакалавра в Санкт-Петербургском государственном университете по направлению Математическое обеспечение и администрирование информационных систем. Ее области научных интересов включают теорию формальных языков и ее применения.

Yuliya Alekseevna SUSANINA received a Bachelor degree in Saint Petersburg State University on Software and Administration of Information Systems programme. Her research interests include formal language theory and its applications.

Анна Никитична ЯВЕЙН - получила степень бакалавра в Санкт-Петербургском государственном университете по направлению Прикладная математика и информатика. Ее области научных интересов включают статический анализ кода и компиляторы.

Anna Nikitichna YAVEYN received a Bachelor degree in Saint Petersburg State University on Applied Mathematics and Computer Science Systems programme. Her research interests include static code analysis and compilers.
Susanina Y.A., Yaveyn A.N., Grigorev S.V. Modification of Valiant's algorithm for the string matching problem. Trudy ISP RAN/Proc. ISP

Семен Вячеславович ГРИГОРЬЕВ - кандидат физико-математических наук, работает в Санкт-Петербургском государственном университете. В 2016 году защитил кандидатскую диссертацию на тему "Синтаксический анализ динамически формируемых программ". Области научных интересов: теория формальных языков и её приложения, графовые базы данных, статический анализ кода, параллельные вычисления.

Semyon Vyacheslavovich GRIGOREV is PhD in Physical and Mathematical Sciences in Sain Petersburg State University. He defended his $\mathrm{PhD}$ thesis «Parsing of dynamically generated code in 2016. His research interests include formal language theory and applications, graph databases, static code analysis and parallel computing. 